Supporting Information for

\title{
Refined Dummy Atom Model of Mg2+ by Simple Parameter Screening Strategy with Revised Experimental Solvation Free Energy
}

\author{
Yang Jiang, Haiyang Zhang, Wei Feng, and Tianwei Tan * \\ Beijing Key Lab of Bioprocess, College of Life Science and Technology, Beijing University of \\ Chemical Technology, Beijing 100029, China \\ *Corresponding author: twtan@mail.buct.edu.cn
}




\section{Test of Saxena's Dummy Model of $\mathrm{Mg}^{2+}$}

In order to evaluate the simulation performance of Saxena's model, we ran a 10-ns MD simulation of $\mathrm{Mg}^{2+}$ ion parameterized by this model in water. All the simulation parameters were same with those in Section 2.4. Unfortunately, the simulation was terminated with an error in the heating step when we used the latest Amber14 version, but without any errors when we used Amber11. The error indicated that, during the simulation, the structure of this $\mathrm{Mg}^{2+}$ dummy model cannot keep stable. Its energy shot up to induce a large velocity of dummy atoms, which was beyond the velocity limitation and finally destroyed the octahedron structure (see Figure S6). We then reran this simulation several times with different initial velocities using Amber14. But the same error occurred in all the simulations. We then used Amber11 to calculate the solvation free energy. But during the perturbation of atomic charge, Amber11 also reported the same error. In addition, according to the result of the only one successful simulation using Amber11, the calculated $\mathrm{Mg}-\mathrm{O}$ distances were around $2.05 \AA$, which is smaller than the experimental value $2.09 \AA$ A. Saxena's model has smaller force constants for bond and angle than other models. In our opinion, these small force constants may sometimes fail to keep the octahedron structure stable. Therefore, the use of Saxena's model needs more cautions than the use of other models. We thus gave up testing this model in the present work.

\section{Computational Details of Evaluating Influence of Different Models}

\subsection{Evaluating Influence of Using Different Models on Nonbonding Potentials}

To investigate the reason why different parameterization models have different simulation performance, a simple test was performed. As shown in Figure S5a, there was only one $\mathrm{Mg}^{2+}$ ion (M) with one coordinated oxygen atom (O). In the first evaluation, the metal ion was built by Åqvist's model, 
Duarte's model and our refined model, respectively; and the atom $\mathrm{O}$ was the oxygen atom of TIP3P water in the AMBER ff03 force field. The vdW potential of atom $\mathrm{O}$ in each model can be calculated by

$$
\left\{\begin{array}{l}
U_{\text {Aquist }}^{\text {vdW }}(r)=\sqrt{\varepsilon_{M}^{\text {Aquist }} \varepsilon_{O}^{A M B E R}}\left[\left(\frac{R_{M}^{\text {Aqvist }}+R_{O}^{A M B E R}}{r}\right)^{12}-2\left(\frac{R_{M}^{\text {Aqvist }}+R_{O}^{A M B E R}}{r}\right)^{6}\right] \\
U_{\text {Duarte }}^{\text {vdW }}(r)=\sum_{i=1}^{7}\left[\frac{A_{M_{i}}^{\text {Duarte }} \sqrt{\varepsilon_{O}^{A M B E R}\left(2 R_{O}^{A M B E R}\right)^{12}}}{r_{i}^{12}}-\frac{B_{M_{i}}^{\text {Duarte }} \sqrt{2 \varepsilon_{O}^{A M B E R}\left(2 R_{O}^{A M B E R}\right)^{6}}}{r_{i}^{6}}\right] \\
U_{\text {refined }}^{\text {vdW }}(r)=\sum_{i=1}^{7}\left\{\sqrt{\varepsilon_{M_{i}}^{\text {refined }} \varepsilon_{O}^{A M B E R}}\left[\left(\frac{R_{M_{i}}^{\text {refined }}+R_{O}^{A M B E R}}{r_{i}}\right)^{12}-2\left(\frac{R_{M_{i}}^{\text {refined }}+R_{O}^{A M B E R}}{r_{i}}\right)\right]\right.
\end{array}\right\},
$$

and the electrostatic potential of atom $\mathrm{O}$ in each model can be calculated by

$$
\left\{\begin{array}{l}
U_{\text {Aqvist }}^{\text {ele }}(r)=332 \frac{Q_{M}^{\text {Aqvist }} Q_{O}^{\text {AMBER }}}{r} \\
U_{\text {Duarte }}^{\text {ele }}(r)=332 \sum_{i=1}^{7}\left(\frac{Q_{M_{i}}^{\text {Duarte }} Q_{O}^{A M B E R}}{r_{i}}\right) \\
U_{\text {refined }}^{\text {ele }}(r)=332 \sum_{i=1}^{7}\left(\frac{Q_{M_{i}}^{\text {refined }} Q_{O}^{A M B E R}}{r_{i}}\right)
\end{array}\right.
$$

where the subscript $i$ represents the different atoms in the dummy model. Notice that, the $U_{\text {Duarte }}^{\text {ele }}$ and $U_{\text {refined }}^{\text {ele }}$ have a same value because of the same atomic charge of each atom in the dummy models.

Thus, the comparison between different $\mathrm{vdW}$ and electrostatic potentials of these different models can be easily performed.

\subsection{Evaluating Influence of Using Duarte's Model in Different Force Fields}

For the influence of different force fields, we compared the total nonbonding potentials of atom $\mathrm{O}$ in OPLS-AA force field and AMBER force field with the metal ion (same testing model described in above section) built in Duarte's model. The total nonbonding potentials of atom $\mathrm{O}$ can be calculated by 


$$
\left\{\begin{array}{l}
U_{O P L S}^{\text {nonbond }}(r)=\sum_{i=1}^{7}\left\{\left[\frac{A_{M_{i}}^{\text {Duarte }} \sqrt{4 \varepsilon_{O}^{\text {OPLS }} \sigma_{O}^{\text {OPLS }}{ }^{12}}}{r_{i}^{12}}-\frac{B_{M_{i}}^{\text {Duarte }} \sqrt{4 \varepsilon_{O}^{O P L S} \sigma_{O}^{\text {OPLS }}}}{r_{i}^{6}}\right]+332 \frac{Q_{M_{i}}^{\text {Duarte }} Q_{O}^{\text {OPLS }}}{r_{i}}\right\} \\
U_{\text {AMBER }}^{\text {nonbond }}(r)=\sum_{i=1}^{7}\left\{\left[\frac{A_{M_{i}}^{\text {Duarte }} \sqrt{\varepsilon_{O}^{A M B E R}\left(2 R_{O}^{A M B E R}\right)^{12}}}{r_{i}^{12}}-\frac{B_{M_{i}}^{\text {Duarte }} \sqrt{2 \varepsilon_{O}^{A M B E R}\left(2 R_{O}^{\text {AMBER }}\right)^{6}}}{r_{i}^{6}}\right]+332 \frac{Q_{M_{i}}^{\text {Duarte }} Q_{O}^{\text {AMBER }}}{r_{i}}\right\} .
\end{array}\right.
$$

The second equation can simply describe the situation that using Duarte's model in AMBER force field through the strategy described in Section 2.1 of the main text. Thus, the relative error $E$ of using Duarte's model in the AMBER ff03 force field can be defined as eq. 12 in the main text. Here, four ligands of $\mathrm{Mg}^{2+}$ (TIP3P-O, Glu-OE1, Thr-OG1 and Asp-OD1; parameters are shown in Table S3) that are commonly observed in metalloenzymes were selected to calculate the different relative errors. All the above calculations were performed in MATLAB. 


\section{Supporting Tables and Figures}

Table S1. Duarte's dummy model parameters

Force Field Parameters

\begin{tabular}{|c|c|c|c|c|}
\hline \multicolumn{2}{|c|}{ Bond type $^{a}$} & \multicolumn{2}{|c|}{$K_{b}$} & $b_{0}$ \\
\hline \multicolumn{2}{|c|}{$\mathrm{MC}-\mathrm{D}$} & \multicolumn{2}{|c|}{800.0} & 0.900 \\
\hline \multicolumn{2}{|c|}{ Angle type ${ }^{b}$} & \multicolumn{2}{|c|}{$K_{\theta}$} & $\theta_{0}$ \\
\hline \multicolumn{2}{|c|}{$\mathrm{D}_{\mathrm{i}}-\mathrm{MC}-\mathrm{D}_{\mathrm{i}}$} & \multicolumn{2}{|c|}{125.0} & 180.0 \\
\hline \multicolumn{2}{|c|}{$\mathrm{D}_{\mathrm{i}}-\mathrm{MC}-\mathrm{D}_{\mathrm{j} \neq \mathrm{i}}$} & \multicolumn{2}{|c|}{125.0} & 90.0 \\
\hline Atom type & mass & charge & $A_{i}^{c}$ & $B_{i}^{c}$ \\
\hline $\mathrm{MC}$ & 6.3 & -1.00 & 63.00 & 9.00 \\
\hline $\mathrm{D}$ & 3.00 & 0.50 & 0.05 & 0.00 \\
\hline
\end{tabular}

${ }^{a} U_{b}=K_{b}\left(b-b_{0}\right)^{2}$, where $K_{b}$ is in $\mathrm{kcal} \mathrm{mol}^{-1} \AA^{-2}$ and $b_{0}$ is in $\AA$;

${ }^{b} U_{\theta}=K_{\theta}\left(\theta-\theta_{0}\right)^{2}$, where $K_{\theta}$ is in $\mathrm{kcal} \mathrm{mol}^{-1} \mathrm{rad}^{-2}$ and $\theta_{0}$ is in degrees;

${ }^{c} A_{i}$ is in $\mathrm{kcal}^{1 / 2} \mathrm{~mol}^{-1 / 2} \AA^{6}$ and $B_{i}$ is in $\mathrm{kcal}^{1 / 2} \mathrm{~mol}^{-1 / 2} \AA^{3}$

Table S2. The threshold values ${ }^{a}$ of different iteration steps

\begin{tabular}{ccc}
\hline & $\mathrm{T} 1(\AA)$ & $\mathrm{T} 2(\AA)$ \\
\cline { 2 - 3 } Iteration I & 0.020 & 0.015 \\
Iteration II & 0.010 & 0.005 \\
\hline
\end{tabular}

${ }^{a}$ The usage of these threshold values T1, T2 and T3 can be found in Fig. 2 in the main text. 
Table S3. Nonbonding parameters of some oxygen atoms in OPLS-AA force field and AMBER force field

\begin{tabular}{ccccccc}
\hline \multirow{2}{*}{ Atom O } & \multicolumn{3}{c}{ OPLS-AA } & & \multicolumn{3}{c}{ AMBER } \\
TIP3P-O & 3.15061 & 0.152 & -0.834 & 1.7683 & 0.152 & -0.834 \\
\cline { 2 - 6 } GLU-OE1 & 2.96 & 0.21 & -0.8 & 1.6612 & 0.21 & -0.824 \\
THR-OG1 & 3.12 & 0.1703 & -0.683 & 1.721 & 0.2104 & -0.6019 \\
ASP-OD1 & 2.96 & 0.21 & -0.8 & 1.6612 & 0.21 & -0.7304 \\
\hline
\end{tabular}

${ }^{a} R$ is the same parameter with $R_{\min } / 2$ and satisfies $R=\frac{1}{2} \sigma \cdot 2^{\frac{1}{6}}$. 
Table S4. Performance of the four different models compared with the QM optimized metal center from 1M0W

\begin{tabular}{ccccc}
\hline & Åqvist & Allnér & Duarte & This work \\
\cline { 2 - 4 }$r^{2}$ Hessian & 0.680 & 0.675 & 0.670 & 0.664 \\
RMSd bond $(\AA)$ & 0.233 & 0.118 & 0.216 & 0.082 \\
RMSd angle (degree) & 21.4 & 8.6 & 15.5 & 7.6 \\
RMSd dihedral (degree) & 105.6 & 80.6 & 106.7 & 60.2 \\
RMSd $(\AA)$ & 0.81 & 0.83 & 0.94 & 0.71 \\
\hline
\end{tabular}

Table S5. Performance of the four different models compared with the QM optimized metal center from 4U5X

\begin{tabular}{ccccc}
\hline & Åqvist & Allnér & Duarte & This work \\
\cline { 2 - 4 }$r^{2}$ Hessian & 0.733 & 0.728 & 0.713 & 0.712 \\
RMSd bond $(\AA)$ & 0.119 & 0.094 & 0.076 & 0.079 \\
RMSd angle (degree) & 4.7 & 4.8 & 5.8 & 5.8 \\
RMSd dihedral (degree) & 90.3 & 90.4 & 92.2 & 92.1 \\
RMSd $(\AA)$ & 0.39 & 0.34 & 0.89 & 0.89 \\
\hline
\end{tabular}


Table S6. Mg-O distances obtained from the simulations in this work for protein 1PYX

\begin{tabular}{|c|c|c|c|c|}
\hline Distance $(\AA)$ & X-ray & Åqvist $^{a}$ & Duarte & This work \\
\hline $\mathrm{Mg}(\mathrm{I}) \cdots$ ATP-O2A & 2.08 & $1.89 \pm 0.04$ & $2.04 \pm 0.04$ & $1.98 \pm 0.04$ \\
\hline $\mathrm{Mg}(\mathrm{I}) \cdots$ ATP-O2G & 1.97 & $1.84 \pm 0.04$ & $2.03 \pm 0.04$ & $1.99 \pm 0.04$ \\
\hline $\mathrm{Mg}(\mathrm{I}) \cdots$ Asp200-OD2 & 2.25 & $1.90 \pm 0.05$ & $2.07 \pm 0.04$ & $2.02 \pm 0.05$ \\
\hline $\mathrm{Mg}(\mathrm{I}) \cdots$ Asn186-OD1 & 2.13 & $2.09 \pm 0.11$ & $2.15 \pm 0.06$ & $2.14 \pm 0.07$ \\
\hline $\operatorname{Mg}(\mathrm{I}) \cdots$ Wat 1 & Not exist ${ }^{b}$ & $2.07 \pm 0.08$ & $2.13 \pm 0.05$ & $2.10 \pm 0.05$ \\
\hline $\operatorname{Mg}(\mathrm{I}) \cdots$ Wat2 & Not exist $^{a}$ & $2.05 \pm 0.07$ & $2.14 \pm 0.05$ & $2.09 \pm 0.05$ \\
\hline Mg (II) $\cdots$ Asp200-OD2 & 2.20 & Break $^{c}$ & Break $^{c}$ & Break $^{c}$ \\
\hline Mg (II) $\cdots$ Asp200-OD1 & 2.23 & $1.90 \pm 0.05$ & $2.09 \pm 0.04$ & $2.03 \pm 0.05$ \\
\hline $\mathrm{Mg}$ (II) $\cdots$ ATP-O2B & 2.15 & $1.88 \pm 0.04$ & $2.06 \pm 0.04$ & $2.02 \pm 0.04$ \\
\hline $\mathrm{Mg}(\mathrm{II}) \cdots$ ATP-O3G & 2.34 & $1.89 \pm 0.05$ & $2.04 \pm 0.04$ & $2.00 \pm 0.04$ \\
\hline Mg (II) $\cdots$ Wat3 & 2.36 & $2.06 \pm 0.08$ & $2.14 \pm 0.05$ & $2.10 \pm 0.05$ \\
\hline Mg (II) $\cdots$ Wat4 & 2.19 & $2.05 \pm 0.07$ & $2.14 \pm 0.05$ & $2.11 \pm 0.05$ \\
\hline $\operatorname{Mg}($ II) $\cdots$ Wat5 & Not exist $^{a}$ & $2.06 \pm 0.07$ & $2.13 \pm 0.05$ & $2.10 \pm 0.05$ \\
\hline
\end{tabular}

${ }^{a}$ The calculated distances for Åqvist's parameter is obtained from ref.6.

${ }^{b}$ "Not exist" means there is no such ligand coordinating with $\mathrm{Mg}^{2+}$.

$c$ "Break" means the ligand was replaced by another molecule and never coordinated with $\mathrm{Mg}^{2+}$ during the simulation. 
Table S7. Mg-O distances obtained from the simulations in this work for protein $1 \mathrm{MOW}$

\begin{tabular}{|c|c|c|c|c|c|c|}
\hline Distance $(\AA)$ & X-ray & Åqvist & Allnér & Duarte & This work & Restrained \\
\hline $\mathrm{Mg}(\mathrm{I}) \cdots$ ATP-O2A & 2.14 & $1.86 \pm 0.04$ & $1.93 \pm 0.04$ & $2.05 \pm 0.04$ & $2.01 \pm 0.04$ & $2.10 \pm 0.05$ \\
\hline $\mathrm{Mg}(\mathrm{I}) \cdots$ ATP-O2G & 2.23 & $1.88 \pm 0.05$ & $1.95 \pm 0.06$ & $2.06 \pm 0.04$ & $2.02 \pm 0.04$ & $2.16 \pm 0.06$ \\
\hline Mg (I) $\cdots$ Glu146-OE1 & 2.13 & $1.86 \pm 0.04$ & $1.96 \pm 0.05$ & $2.06 \pm 0.04$ & $2.02 \pm 0.04$ & $2.06 \pm 0.06$ \\
\hline $\operatorname{Mg}(\mathrm{I}) \cdots$ Wat 1 & 2.19 & $2.07 \pm 0.08$ & $2.10 \pm 0.08$ & $2.13 \pm 0.05$ & $2.10 \pm 0.05$ & $2.18 \pm 0.07$ \\
\hline $\operatorname{Mg}(\mathrm{I}) \cdots$ Wat2 & 2.17 & $2.02 \pm 0.08$ & $2.09 \pm 0.08$ & $2.13 \pm 0.05$ & $2.10 \pm 0.05$ & $2.16 \pm 0.07$ \\
\hline $\operatorname{Mg}(\mathrm{I}) \cdots$ Wat3 & 2.24 & Changing $^{a}$ & Changing $^{a}$ & $2.13 \pm 0.05$ & $2.09 \pm 0.05$ & $2.20 \pm 0.07$ \\
\hline Mg (II) ‥ Glu146-OE1 & 2.44 & Break $^{b}$ & Break $^{b}$ & Break $^{b}$ & Break $^{b}$ & $2.45 \pm 0.09$ \\
\hline Mg (II) $\cdots$ Glu146-OE2 & 2.28 & $1.88 \pm 0.04$ & $1.96 \pm 0.05$ & $2.07 \pm 0.04$ & $2.03 \pm 0.04$ & $2.15 \pm 0.08$ \\
\hline Mg (II) $\cdots$ Glu386-OE1 & 2.39 & $1.94 \pm 0.06$ & $2.02 \pm 0.08$ & $2.06 \pm 0.04$ & $2.01 \pm 0.04$ & $2.39 \pm 0.07$ \\
\hline Mg (II) $\cdots$ ATP-O1B & 2.24 & $1.89 \pm 0.05$ & $1.96 \pm 0.05$ & $2.07 \pm 0.04$ & $2.03 \pm 0.04$ & $2.19 \pm 0.06$ \\
\hline Mg (II) $\cdots$ ATP-O1G & 2.19 & $1.86 \pm 0.04$ & $1.94 \pm 0.05$ & $2.07 \pm 0.04$ & $2.02 \pm 0.04$ & $2.13 \pm 0.06$ \\
\hline Mg (II) $\cdots$ Wat4 & 2.34 & $2.11 \pm 0.09$ & $2.11 \pm 0.08$ & $2.13 \pm 0.05$ & $2.10 \pm 0.05$ & $2.29 \pm 0.10$ \\
\hline Mg (II) $\cdots$ Wat5 & Not exist ${ }^{c}$ & Changing $^{a}$ & Changing $^{a}$ & $2.13 \pm 0.05$ & $2.09 \pm 0.05$ & Not exist ${ }^{c}$ \\
\hline Mg (II) ‥ Glu386-OE2 & Not exist $^{c}$ & Changing $^{a}$ & Changing $^{a}$ & Not exist ${ }^{c}$ & Not exist $^{c}$ & Changing $^{a}$ \\
\hline
\end{tabular}

a "Changing" means the ligand did not always coordinated with $\mathrm{Mg}^{2+}$. The coordination state was changing during the simulation.

$b$ “Break" means the ligand was replaced by another molecule during the simulation.

$c$ "Not exist" means there is no such ligand coordinating with $\mathrm{Mg}^{2+}$. 
Table S8. Mg-O distances obtained from the simulations in this work for protein 4 U5X

\begin{tabular}{cccccc}
\hline Distance $(\AA)$ & X-ray & Åqvist & Allnér & Duarte & This work \\
\cline { 2 - 5 } $\mathrm{Mg} \cdots$ GTP-O2B & 2.00 & $1.87 \pm 0.04$ & $1.96 \pm 0.04$ & $2.06 \pm 0.04$ & $2.02 \pm 0.04$ \\
$\mathrm{Mg} \cdots$ GTP-O2G & 1.97 & $1.81 \pm 0.03$ & $1.89 \pm 0.04$ & $2.03 \pm 0.03$ & $1.98 \pm 0.04$ \\
$\mathrm{Mg} \cdots$ Thr24-OG1 & 2.03 & $2.11 \pm 0.08$ & $2.16 \pm 0.08$ & $2.17 \pm 0.05$ & $2.13 \pm 0.06$ \\
$\mathrm{Mg} \cdots$ Thr42-O & 2.15 & $2.02 \pm 0.07$ & $2.10 \pm 0.07$ & $2.14 \pm 0.05$ & $2.10 \pm 0.05$ \\
$\mathrm{Mg} \cdots$ Wat1 & 2.05 & $2.02 \pm 0.06$ & $2.06 \pm 0.06$ & $2.13 \pm 0.04$ & $2.09 \pm 0.05$ \\
$\mathrm{Mg} \cdots$ Wat2 & 2.06 & $2.02 \pm 0.06$ & $2.06 \pm 0.06$ & $2.12 \pm 0.04$ & $2.08 \pm 0.05$ \\
\hline
\end{tabular}



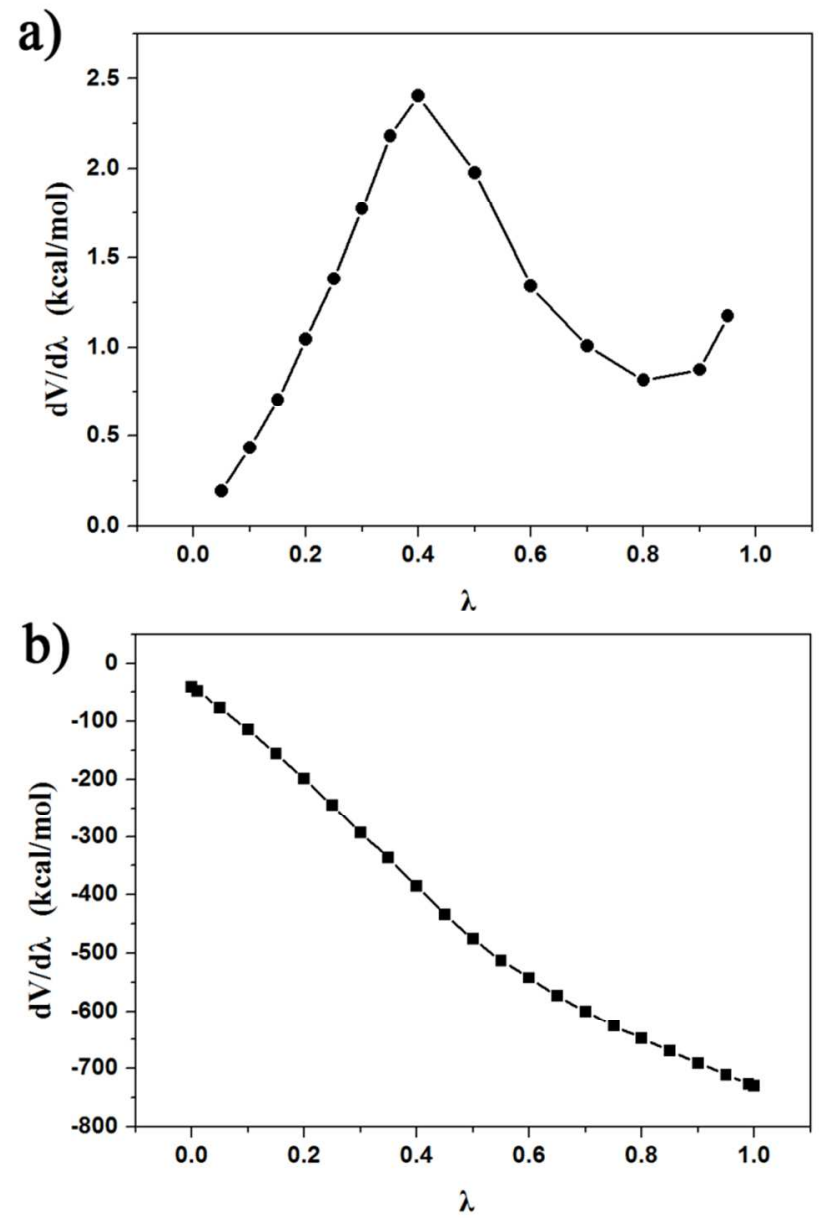

Figure S1. The $d V / d \lambda$ vs. $\lambda$ in the free energy calculation of our final model

a) The $d V / d \lambda$ plot for the vdW perturbation of our final refined model; b) The $d V / d \lambda$ plot for the charge perturbation of our final refined model. The other systems show similar landscapes. 


\section{a) $1 \mathrm{PYX}$}

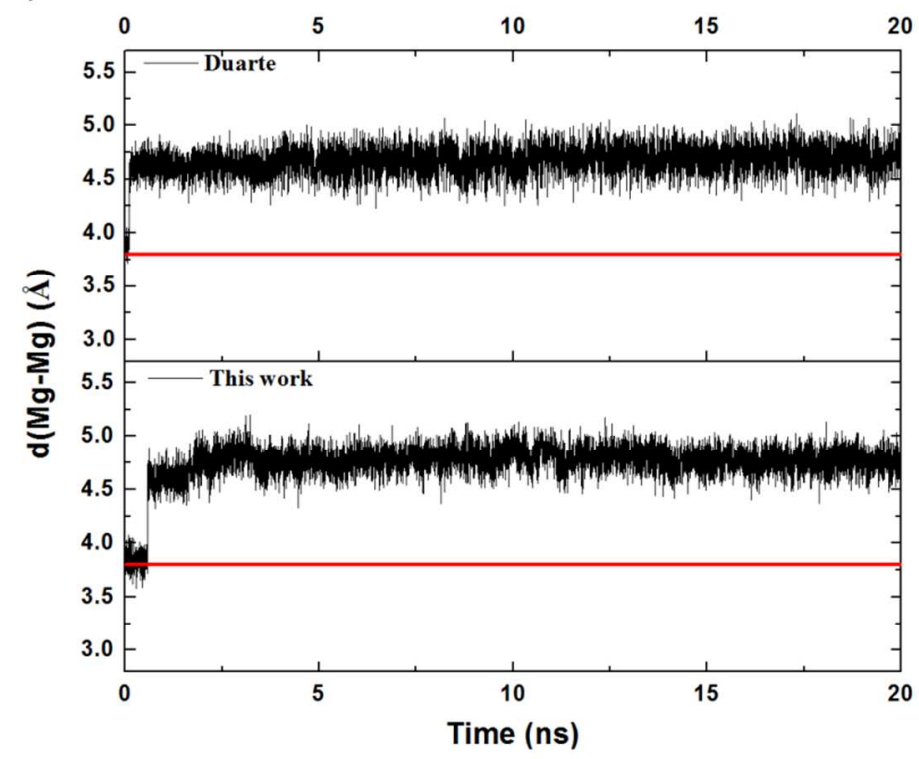

\section{b) $1 \mathrm{M} 0 \mathrm{~W}$}

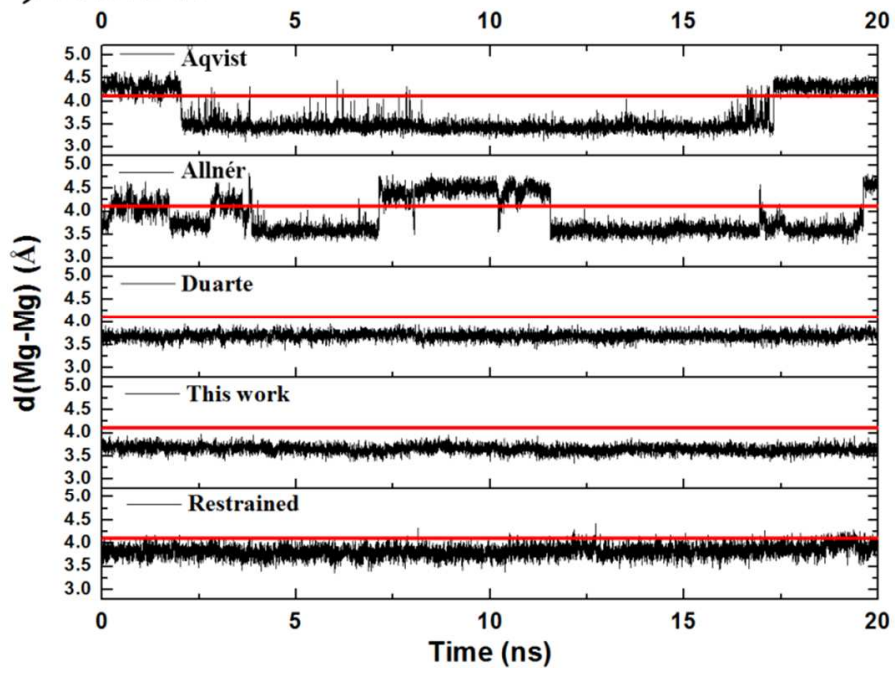

Figure S2. The distance between two $\mathrm{Mg}^{2+}$ in the double-metal center vs. simulation time

a) The Mg-Mg distances for protein 1PYX simulated using Duarte's model and our refined model, respectively. b) The $\mathrm{Mg}-\mathrm{Mg}$ distances for protein 1M0W simulated using Åqvist's model, Allnér's model, Duarte's model, our refined model and the restrained model, respectively. The red line in each subfigure represents the $\mathrm{Mg}-\mathrm{Mg}$ distance obtained from each crystal structure $(3.8 \AA$ for $1 \mathrm{PYX}$ and 4.1 $\AA ̊$ for $1 \mathrm{M} 0 \mathrm{~W})$ 


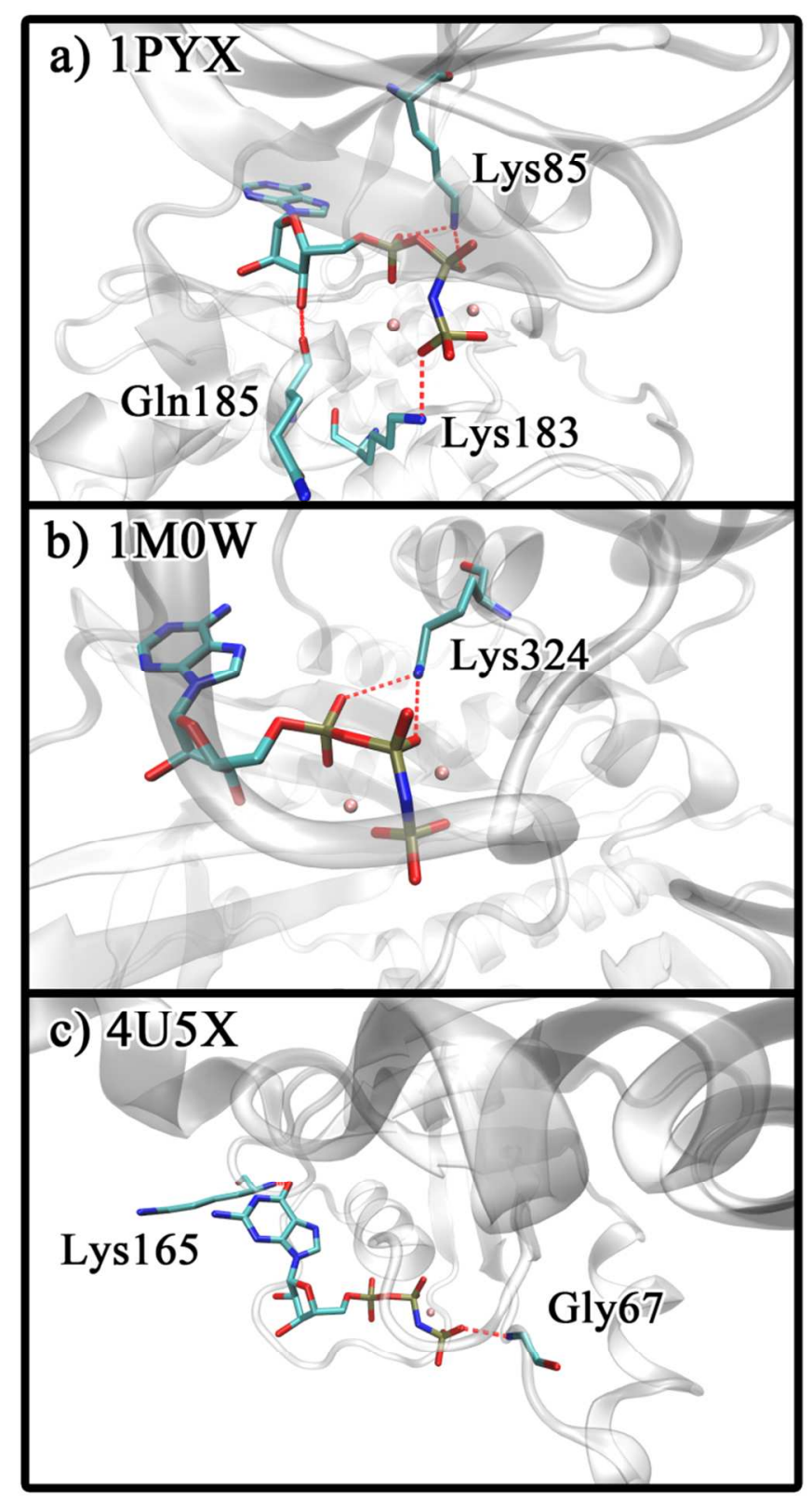

Figure S3. The discussed hydrogen bonds in different protein-cofactor systems

Only the hydrogen bonds that have significant difference between different models and discussed in Section 3 are shown in this figure (subfigure a) for protein 1PYX, b) for protein $1 \mathrm{M} 0 \mathrm{~W}$ and c) for protein 4U5X). Proteins are shown in transparent white. Hydrogen bonds are represented by red dashed 
lines. The residues that form these hydrogen bonds are marked. $\mathrm{Mg}^{2+}$ ions are shown as pink spheres. Each structure is obtained from its crystal structure.

a)

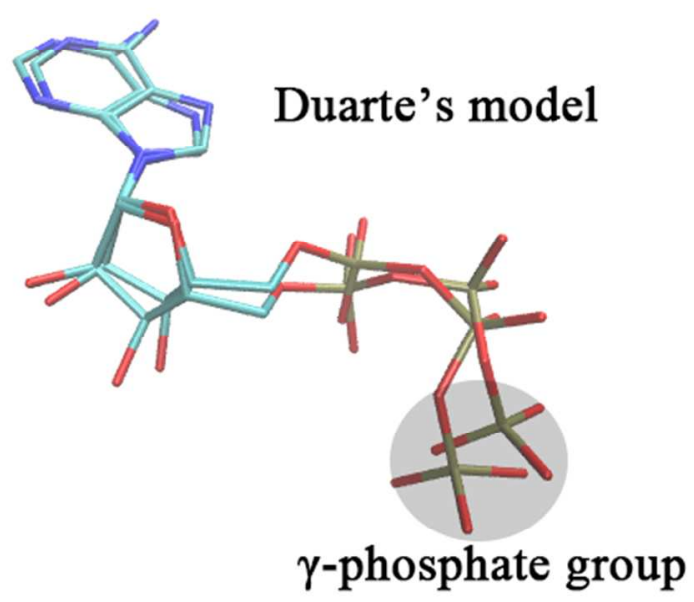

b)

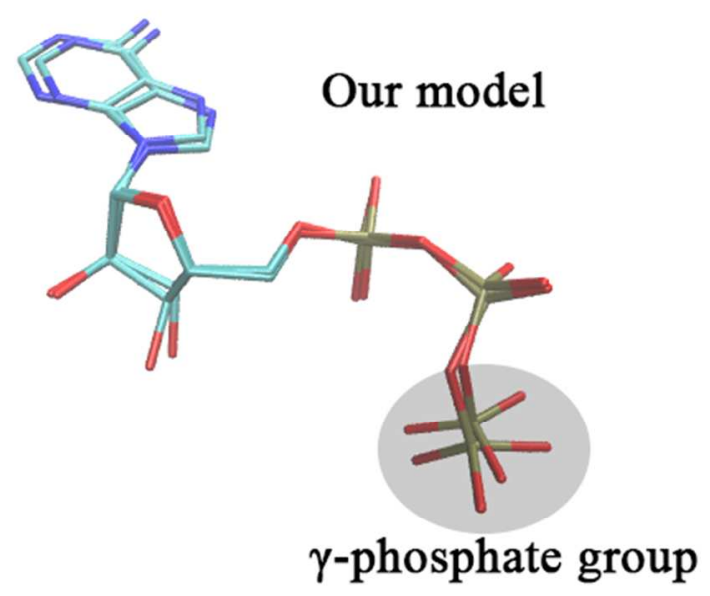

Figure S4. The comparison of the simulated structure and the crystal structure of ATP molecule in protein 1PYX

a) The ATP was simulated in the system with $\mathrm{Mg}^{2+}$ parameterized by Duarte's model. b) The ATP was simulated in the system with $\mathrm{Mg}^{2+}$ parameterized by our refined model. The simulated structure is obtained from the average structure of each simulation and superposed with the crystal structure. The $\gamma$-phosphate group of ATP is marked by a gray ellipse. Large structural deviations of the simulated ATP molecule for Duarte's model can be observed. 
a)

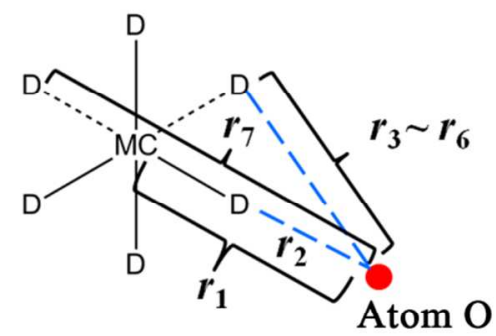

c)

$$
r_{i}= \begin{cases}r & i=1 \\ r-b & i=2 \\ \sqrt{r^{2}+b^{2}} & i=3,4,5,6 \\ r+b & i=7\end{cases}
$$

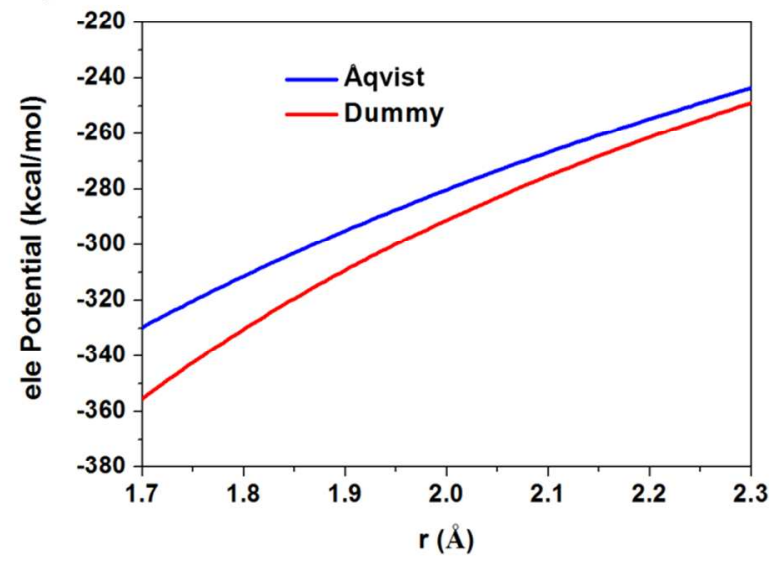

b)

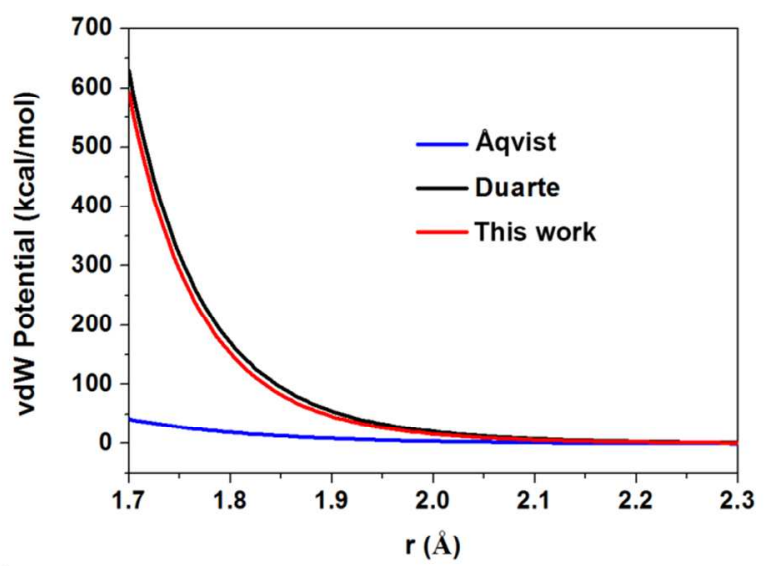

d)

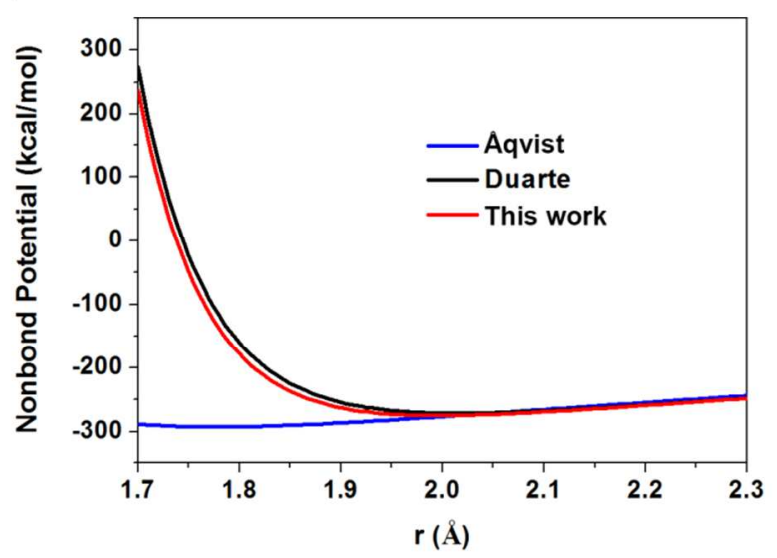

Figure S5. The comparison of the nonbonding potentials of each model

a) The diagram of the distance between atom $\mathrm{O}$ (red sphere) and the dummy model $\mathrm{Mg}^{2+}$ ion. The seven distances can be represented by the distance $r$ between the metal core (MC) and atom $\mathrm{O}$; and the distance $r$ is also the distance between $\mathrm{Mg}^{2+}$ and atom $\mathrm{O}$ for Aqquist's model. $b$ is the bond length between MC and dummy atoms (D), and equals $0.9 \AA$. b) The vdW potential of the atom O vs. the distance $r$. c) The electrostatic potential of the atom $\mathrm{O}$ vs. the distance $r$. d) The total nonbonding potential of the atom O vs. the distance $r$. Only the distances between $1.7 \AA$ and $2.3 \AA$ are shown in the figure, because the distance value usually appears in this range during the simulations of $\mathrm{Mg}^{2+}$-water system. The Åqvist's model, Duarte's model and our refined model are represented by blue, black and 
red curves, respectively.
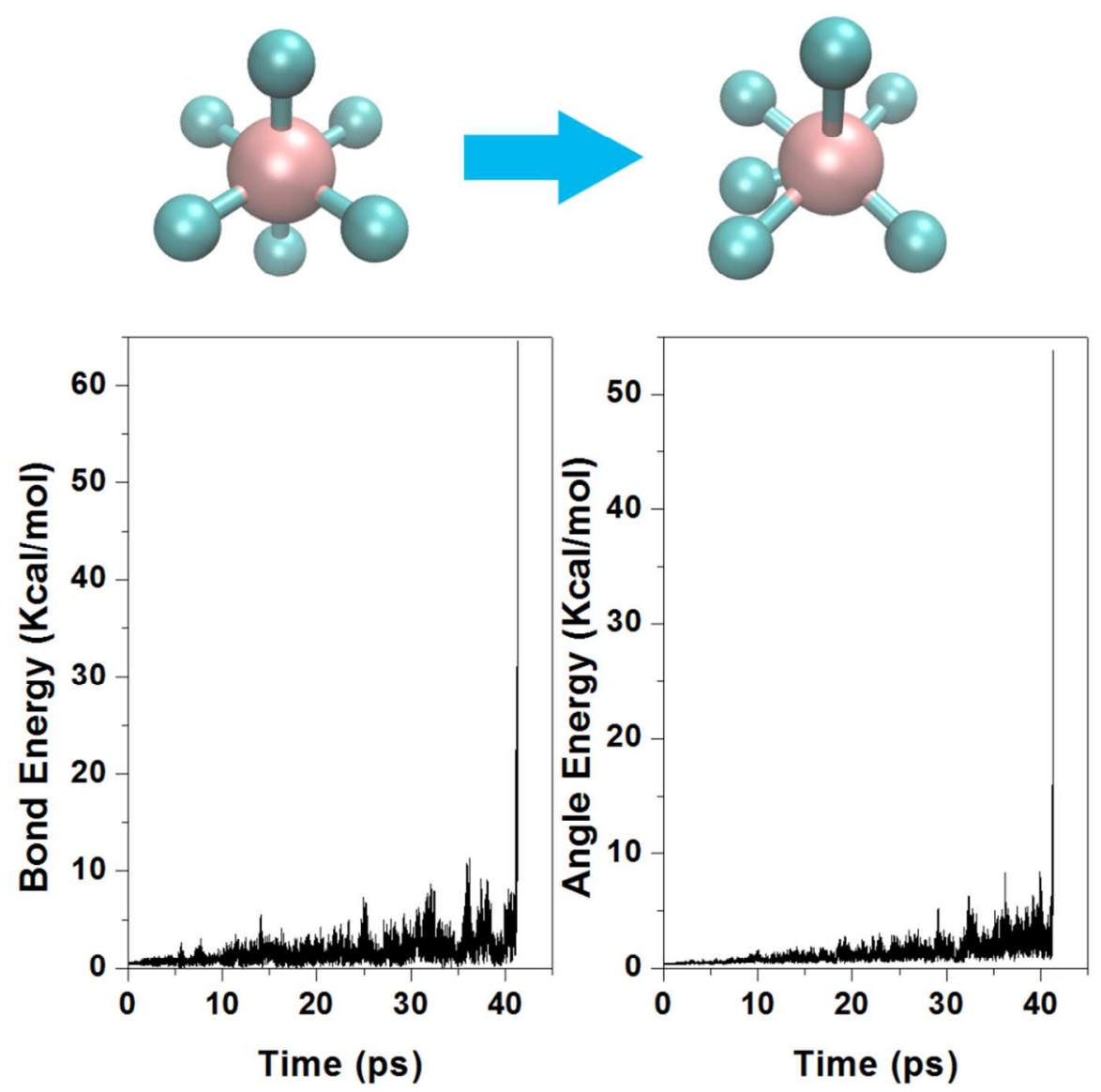

Figure S6. The structure and the energies of bond and angle of Mg2+ modeled using Saxena's parameters in our failed simulation

We can see a serious conformational change of dummy model during 60-ps heating process. The bond energy and angle energy shoot up, and the simulation is terminated at $41.3 \mathrm{ps}$. 